\title{
Temporal modulation transfer functions in the European Starling (Sturnus vulgaris): II. Responses of auditory-nerve fibres
}

\author{
O. Gleich * , G.M. Klump \\ Institut für Zoologie der Technischen Universität München, Lichtenbergstrasse 4, 85747 Garching, FRG
}

(Received 9 April 1994; revision received 5 October 1994; accepted 19 October 1994)

\begin{abstract}
The temporal resolution of cochlear-nerve fibres in the European starling was determined with sinusoidally amplitude-modulated noise stimuli similar to those previously used in a psychoacoustic study in this species (Klump and Okanoya, 1991). Temporal modulation transfer curves (TMTFs) were constructed for cochlear afferents allowing a direct comparison with the starling's behavioural performance. On average, the neuron's detection of modulation was less sensitive than that obtained in the behavioural experiments, although the most sensitive cells approached the values determined psychophysically. The shapes of the neural TMTFs generally resembled low-pass or band-pass filter functions, and the shapes of the averaged neural functions were very similar to those obtained in the behavioural study for two different types of stimuli (gated and continuous carrier). Minimum integration times calculated from the upper cut-off frequency of the neural TMTFs had a median of $0.97 \mathrm{~ms}$ with a range of 0.25 to $15.9 \mathrm{~ms}$. The relations between the minimum integration times and the tuning characteristics of the cells (tuning curve bandwidth, $Q_{10} \mathrm{~dB}$-value, high- and low-frequency slopes of the tuning curves) are discussed. Finally, we compare the TMTF data recorded in the starling auditory nerve with data from neurophysiological and behavioural observations on temporal resolution using other experimental paradigms in this and other vertebrate species.
\end{abstract}

Keywords: Temporal resolution; Amplitude modulation; Auditory nerve; Bird; Hearing

\section{Introduction}

Sufficient temporal resolution of the auditory system forms the basis of our ability to perceive the rapid amplitude changes that are characteristic for many speech signals and may be essential for their perception (e.g., see Fastl et al., 1986; Palmer 1990; Winter and Palmer, 1990). Thus, the measurement of temporal resolution is considered an important issue in the study of acoustic perception. Two experimental paradigms have been applied to study the temporal resolution of the auditory system. In the first paradigm (gap-detection), the auditory system's ability to report the occurrence of a brief single drop in the envelope of the signal is measured (e.g., see Shailer and Moore, 1983,). In the second paradigm (temporal modulation transfer

\footnotetext{
${ }^{*}$ Corresponding author. (Present address): HNO-Klinik, Universität Regensburg, Postfach 93042, Regensburg, FRG. Fax: + 49 (941) 9449402.
}

functions), the trade-off between the frequency of a periodic sinusoidal amplitude modulation and the depth of modulation at the modulation-detection threshold is determined (e.g., see Viemeister, 1979). In both paradigms, a broad-band (white) noise is used as the carrier signal to eliminate use of spectral cues by the subjects in the detection of the change in the envelope of the signal. Typically, the temporal modulation transfer functions have a low-pass or band-pass characteristic, and the minimum integration time can be derived from the upper cut-off frequency of this function as a parameter that describes the auditory system's temporal resolution. In the auditory systems studied so far with both paradigms using psychoacoustic methods, the minimum integration time is about half the duration of the minimum-detectable gap (human: see Formby and Muir, 1988, for a review; chinchilla: see Henderson et al., 1984 and Giraudi et al., 1980; European starling: Klump and Maier, 1989, and Klump and Okanoya, 1991).

Temporal resolution in the neuronal coding of the stimulus envelope generally is lower, the higher the 
anatomical level within the auditory system (for a recent review, see Langner, 1992); European starlings may be an exception of the rule in that primary-like neurons in the auditory forebrain when studied in a gap-detection paradigm show a temporal resolution which is similar to that in the auditory nerve (see Klump and Gleich, 1991). The highest temporal resolution is generally found in the auditory periphery, i.e., in the auditory nerve. Only the information on stimulus patterns that are encoded in the discharges of auditory-nerve fibres can be extracted by the hearing system. Thus, the auditory-nerve forms the first filter in the analysis that may influence the perceptual limits measured in psychoacoustic experiments. For this reason, researchers have studied the temporal resolution of auditory-nerve fibres using the paradigms that have been applied in psychoacoustical studies. Gap-detection using broad-band noise carrier signals has been studied in such a diverse collection of species as an Arctiid moth (Surlykke et al., 1988), a field cricket (Ronacher and Römer, 1985), the goldfish (Fay, 1985), chinchilla (Zhang et al., 1990), and the European starling (Klump and Gleich, 1991). So far, only one study in the coqui frog (Dunia and Narins, 1989) and another in the goldfish (Fay, 1980) have measured temporal resolution using modulation transfer functions determined with a broad-band noise as the carrier. Although such modulation transfer functions have commonly been used in behavioural studies in birds and mammals (e.g. see refs. in Klump and Okanoya, 1991) physiological measurements using the same type of stimulus are not available for comparison.

This study in the European starling (Sturnus vulgaris) measures temporal resolution of auditory-nerve fibres by using temporal modulation transfer functions and broad-band noise as the carrier. It uses stimuli that are similar to those applied by Klump and Okanoya (1991) in measuring temporal resolution psychoacoustically. This will allow a direct comparison of the psychoacoustic performance of the whole animal with that of its peripheral auditory system. Furthermore, the minimum integration time obtained in this study can be compared with other measures of temporal resolution in this species that have previously been determined using the gap detection paradigm (auditory-nerve: Klump and Gleich, 1991; forebrain: Buchfellener et al. 1989; psychoacoustics: Klump and Maier, 1989). Including the results of this study, the starling is the animal model in auditory research in which the most comprehensive data sets on different aspects of temporal resolution is available.

\section{Methods}

The activity of primary auditory afferents was recorded in wild caught starlings (Sturnus vulgaris) from the VIIIth nerve where it entered the brain stem (Klump and Gleich, 1991; Gleich, 1994). The care and use of the animals reported on in this study were according to German laws. Birds were anaesthetized by an initial dose of $20 \mathrm{mg} / \mathrm{kg}$ xylacine (Rompun, Bayer) and $40 \mathrm{mg} / \mathrm{kg}$ ketamine (Ketanest, Parke Davis). Supplementary anaesthetics ( $50 \%$ of the initial dose) were given as necessary, on average once every hour. Respiration was not affected by this regimen so that artificical respiration of the birds was not necessary. The core temperature was kept at $40^{\circ}$ by a regulated heating system. A small screw was glued onto the skull with dental cement. This allowed a stable attachment of the head to a holding device. The auditory nerve was exposed after opening the skull and aspiration of part of the cerebellum. Glass microelectrodes filled with $3 \mathrm{M} \mathrm{KCl}$ and a final resistance of $20-100 \mathrm{M} \Omega$ were placed on the surface of the VIIIth nerve under visual control. The electrode was connected to a WPI 767 electrometer and the signal was fed to an audiomonitor for acoustic monitoring of the neural activity and to an oscilloscope for visual control. On the oscilloscope, the level of a Schmidt trigger for spike discrimination could be monitored and adjusted. The output of the Schmidt trigger was then fed to a custom built computer board for processing and storage of the action potentials.

A closed sound system consisting of a Beyer DT 770 speaker and a Brüel \& Kjaer $1 / 2$ inch microphone with probe tube extension was fit tightly into the ear canal of the bird. The frequency response of the sound system was determined for each experiment and the output was flat within $\pm 4 \mathrm{~dB}$ between 0.1 and $5.0 \mathrm{kHz}$. After electrode and speaker placement the bird was transferred into an electrically-shielded sound-attenuating room. By advancing the electrode with a combination of a hydraulic- and a piezoelectric-microdrive through the auditory nerve single cells were isolated.

Upon encountering a cell it was characterized by recording the response to a matrix of $50 \mathrm{~ms}$ test stimuli covering a range of frequencies ( $2-3$ octaves in $0.1-0.2$ octave steps, including the most sensitive frequency) and sound pressure levels (typically 10-90 dB SPL in 8 $\mathrm{dB}$ steps). Each stimulus was presented three times and the repetition rate was 4 stimuli per second. The generation of the stimuli and the recording of the neural responses was controlled by a custom built computer board including a special software. Isorate contours were calculated from these response matrices and the curve with a response criterion slightly above spontaneous rate (on average $20 \%$ ) was taken as the threshold tuning curve (Gleich and Narins, 1988). The characteristic frequency (CF; the most sensitive frequency), threshold at $\mathrm{CF}$, the bandwidth $10 \mathrm{~dB}$ above CF-threshold, the $Q_{10}$ - $\mathrm{dB}$ value (CF divided by the 
bandwidth $10 \mathrm{~dB}$ above CF-threshold) and the slopes of the low- and high-frequency flanks of the tuning curve were measured from the threshold tuning curves. The spontaneous discharge rate was obtained by averaging the responses to 10 low level test stimuli that were outside the response area of the cell resulting in a total sample of 1.5 seconds.

To characterize the neural response to sinusoidally amplitude modulated noise we used a subset of stimuli that had been used in a previous behavioural investigation of temporal modulation transfer functions in the starling (Klump and Okanoya, 1991). The stimuli consisted of sinusoidally modulated noise with a range of modulation frequencies $(5,20,80,320$ and $1280 \mathrm{~Hz})$ and depth of modulations $(0,12.5,25,50$ and $100 \%)$, so that a total of 21 different combinations of the modulation parameters were tested. Depth of modulation in percent was defined by $\mathrm{m}=100 *\left(\mathrm{~V}_{1}-\right.$ $\left.V_{2}\right) /\left(V_{1}+V_{2}\right)$ with $V_{1}$ and $V_{2}$ being the peak and trough amplitudes respectively (Dooling and Searcy, 1981). Two different types of noise stimuli were used: (1) the gated stimulus type consisted of a 780-ms noise pulse with an 8-ms rise and fall time that was repeated every 1.6 seconds and (2) the continuous stimulus type consisted of 1.6 seconds of continuous noise where only the part that corresponded to the $780-\mathrm{ms}$ noise pulse in the gated stimulus was sinusoidally modulated. These stimuli were presented from a prerecorded tape at a sound pressure level of $75 \mathrm{~dB}$ SPL. Fig. 1 shows examples of an oscillogram (a) and the spectrum (b) of the amplitude modulated portion of the stimulus as it was delivered by the tape recorder. The spectra were obtained by averaging the 10 stimulus presentations that were used in the actual experiments. The spectrum was flat to $\pm 4 \mathrm{~dB}$ above $40 \mathrm{~Hz}$, and no spectral components were evident at the modulation frequency. When the tuning curve of a cell had been measured, then the response to 10 repetitions of either the 21 gated or the 21 continuous noise stimuli were recorded. Only data sets with 10 complete stimulus repetitions were included in the subsequent analyses. If the recording remained stable then the response to the other type of stimulus (pulsed or continuous) was recorded.

Further analysis of the modulation data was performed off-line. For each stimulus period histograms of the 10 presentations, with a fundamental frequency corresponding to the modulation frequency, were constructed from $600-\mathrm{ms}$ samples of the modulated response, starting at the onset of the noise pulse or the modulation of the noise respectively. The bin width of the period histograms was set to 22.5 degrees, because the temporal resolution of the spike acquisition $(50 \mu \mathrm{s})$ did not allow a smaller bin width at the highest modulation frequency $(1280 \mathrm{~Hz})$. From the period histograms the vector strength (VS, length of the normal-
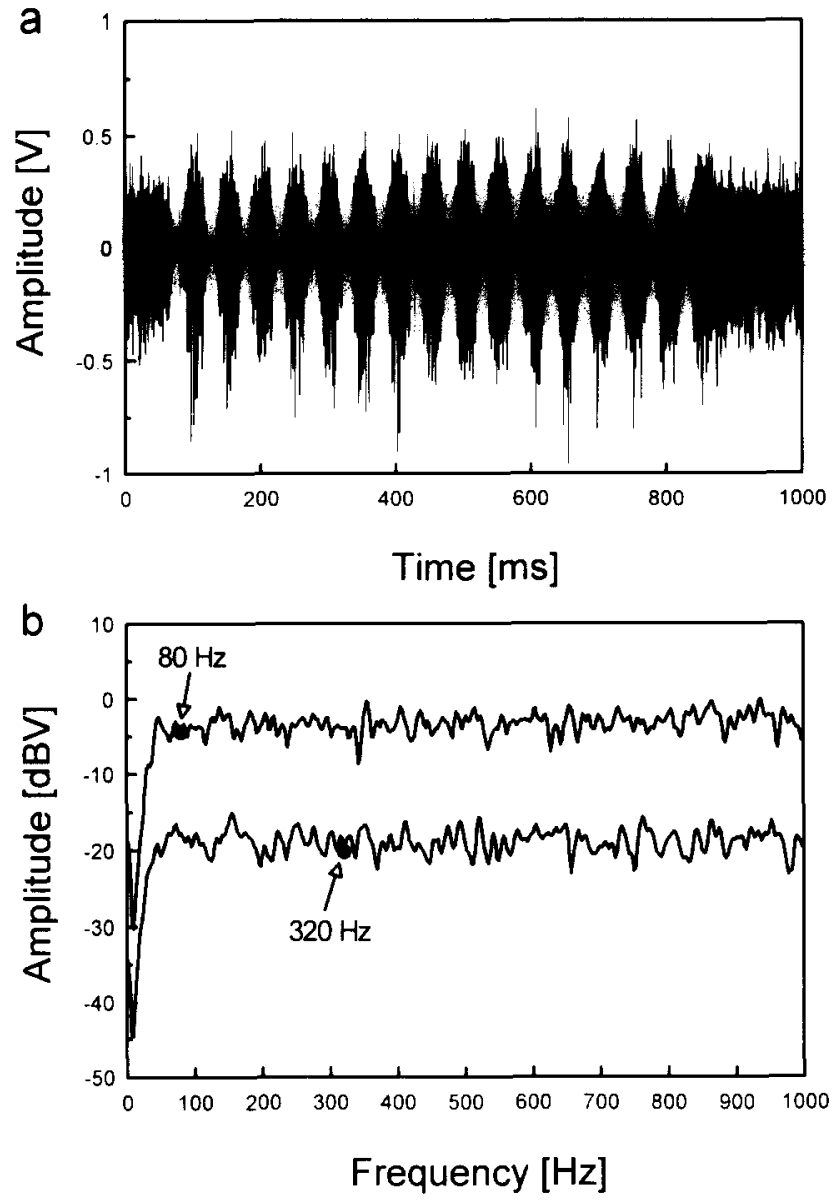

Fig. 1. (a) Example of the time signal of one stimulus $(20 \mathrm{~Hz}$ modulation of a continuous noise, $100 \%$ depth of modulation) as it was delivered by the tape recorder. The time scale starts $50 \mathrm{~ms}$ before the onset of modulation. (b) Examples of the averaged spectra of the modulated signals for modulation frequencies of $80 \mathrm{~Hz}$ and $320 \mathrm{~Hz}$ generated from the 10 repetitions of the gated stimulus presented to the bird. The spectrum of the $320 \mathrm{~Hz}$ amplitude modulated stimulus is shifted down by $15 \mathrm{~dB}$ for clarity.

ized sum vector) and Rayleighs' $\mathrm{z}\left(\mathrm{VS}^{2} * \mathrm{n} ; N=\right.$ the number of spikes) were calculated. A value of 3 for Rayleighs' $\mathrm{z}$ was selected as the threshold criterion for phase locking to the modulation frequency, because for spike numbers of 200 and above this $z$-value corresponds to a significant phase locking at the $5 \%$ level (Zar 1984). Furthermore, this z-value is more than two standard deviations above the mean value determined in the neurons' response to the unmodulated stimulus (which had a mean z-value of 0.80 and a standard deviation of 1.08) For each stimulus frequency Rayleighs' $\mathrm{z}$ was plotted versus depth of modulation (in $\%$ ) and the depth where $\mathrm{z}$ reached a value of 3 was calculated. This value was accepted as modulation threshold, if $z$ remained larger than 3 for further increases in depth of modulation. Temporal modulation transfer (TMTF) curves were then constructed by plotting the modulation thresholds (in $\mathrm{dB}$; $20 * \log (\mathrm{m} / 100) ; \mathrm{m}=$ depth of modulation in $\%)$ as a 
function of the modulation frequency. From the TMTF-functions the cut-off frequency (fc) on the high frequency side was calculated as the frequency where the curve reached a value of $3 \mathrm{~dB}$ below the minimum modulation threshold and the minimum integration time $(1 / 2 * \mathrm{fc})$ was derived from the cut-off frequency.

Statistical analysis of the data was performed using the SPSS for Windows statistics software package.

\section{Results}

TMTF data were obtained from a total of 44 cells in 10 starlings. In 30 cells data for continuous stimuli are available, in 27 of these cells we also have data on the tuning characteristics. In 38 cells the responses to the gated stimuli were recorded. Complete tuning and TMTF data for both gated and continuous stimuli were determined in 21 cells. The distribution of thresholds, $Q_{10} \mathrm{~dB}$ values, slopes of the tuning curve flanks and the spontaneous discharge rates of the fibres in this study resembled those of previous descriptions of starling auditory-nerve fibre characteristics (Manley et al.,
1985; Klump and Gleich, 1991; Gleich, 1994). Similar to the results of these previous studies we found a spread of thresholds between 4 and 80dB SPL. The distribution of spontaneous discharge rates was unimodal with a range between 15 and 142 and a mean of 54 spikes per second.

Fig. 2 shows the response of a typical unit to the 20 $\mathrm{Hz}$ modulation together with oscillograms of the stimulus for presentation with a gated carrier and with a continuous carrier. When presented with the gated carrier, the cell shows a phasic onset response that is followed by a tonic response during which the neuron displays further adaptation. After a short offset inhibition of about $50 \mathrm{~ms}$, the neuron resumes its spontaneous activity. Encoding of each individual cycle of modulation is clearly shown at a depth of modulation of $100 \%$; at times of low stimulus amplitudes, all activity ceases. This cell shows significant phase-locking to the modulation at a minimum depth of modulation of $25 \%$ when the period histograms are analysed.

Typical examples of period histograms constructed from the responses of one cell are shown in Fig. 3. The rows are arranged according to increasing modulation



Fig. 2. Dot-raster plots of the neural responses to the amplitude modulated stimuli by a typical unit (oscillogram of 20 -Hz amplitude modulated stimulus with dot-raster plot below; numbers indicate the depth of modulation; total duration of the time record 1 s). In the top panel, The neuron's response is shown for the gated stimulus; in the bottom panel the response to the continuous noise stimulus is shown. The neuron's $\mathrm{CF}$ was $1.7 \mathrm{kHz}$ and its threshold at CF was $47 \mathrm{~dB}$ SPL. Its spontaneous activity was 19 spikes per second. 


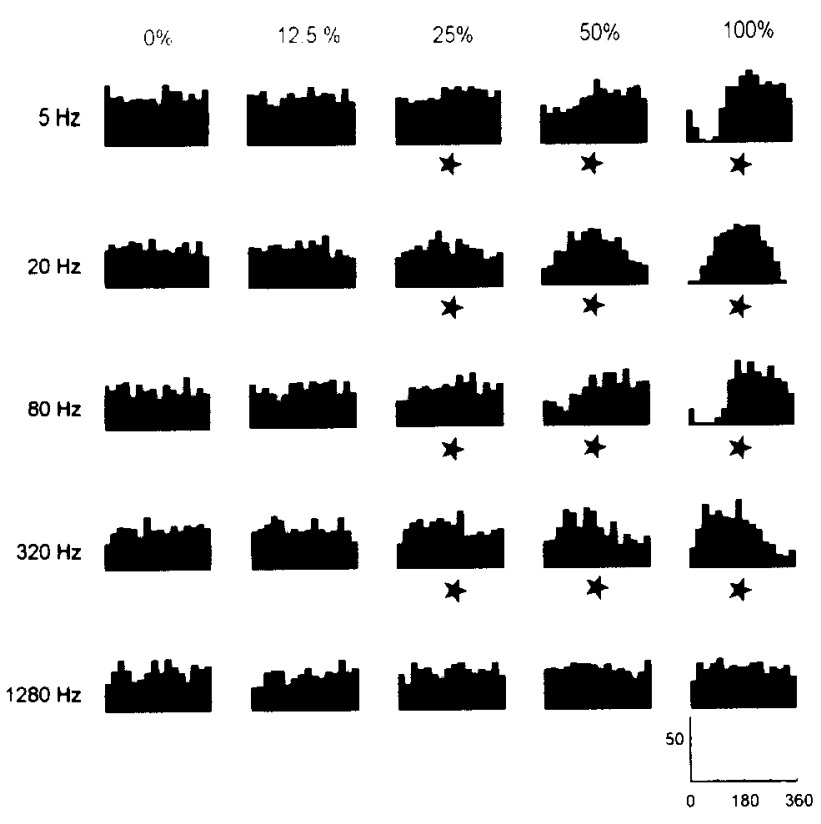

Fig. 3. Period histograms of the responses of an auditory-nerve fibre in relation to the modulation frequency and the depth of modulation when presented with modulations in a continuous carrier. All period histograms are drawn to identical scale with the $\mathrm{x}$-axis ranging from 0 to 360 degrees and the $y$-axis showing the number of spikes per bin (see bottom right). Stars indicate period histograms with significant phase-locking to the modulation ( $P<0.05$, Rayleigh-test). Binwidth of the histograms is 22.5 degrees. The data are from the same neuron as in Fig. 2.

frequency from top to bottom and the columns show increasing depth of modulation from left to right. Strong phase-locking is evident at high depth of modulations and at the 4 lower modulation frequencies. Phase-locking is not seen at a depth of modulation of $0 \%$ and of $12.5 \%$ and at a modulation frequency of $1280 \mathrm{~Hz}$.

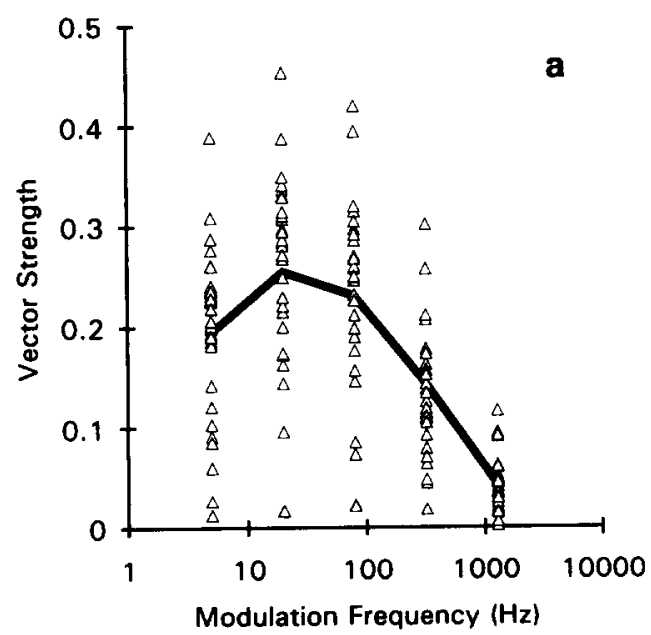

To perform a quantitative analysis of the neuron's responses, the VS and Rayleighs' $\mathrm{z}$ were calculated from such period histograms. In Fig. 4a VS is shown for $100 \%$ depth of modulation as a function of the modulation frequency for the 30 cells tested with the continuous stimuli. The distribution shows a maximum around a modulation frequency of $20 \mathrm{~Hz}$ with VS declining towards lower and higher frequencies. In addition, there is a considerable inter-cell variability of the VS at each modulation frequency. Fig. $4 \mathrm{~b}$ shows the distribution of Rayleighs' $z$ for the same cells and stimuli. There is also a maximum around $20 \mathrm{~Hz}$. At modulation frequencies of $5,20,80$ and $320 \mathrm{~Hz}$ in most cells Rayleighs' $z$ exceeds the critical value of 3 that indicates significant phase-locking. Although most cells show significant phase locking at $320 \mathrm{~Hz}$, Rayleighs' z at this modulation frequency is on average considerably smaller than at 5,20 and $80 \mathrm{~Hz}$. At a modulation frequency of $1280 \mathrm{~Hz}$ only few cells show significant phase locking despite a depth of modulation of $100 \%$.

The value of Rayleighs' $z$ as a function of depth of modulation is shown in Fig. 5a-e for the 5 modulation frequencies. The data are from the same cell as illustrated in Fig. 2 and 3. At modulation frequencies of 5, 20,80 and $320 \mathrm{~Hz}$ Rayleighs' $\mathrm{z}$ increases with the depth of stimulus modulation. However, at $320 \mathrm{~Hz}$ the function is clearly shallower than at the lower modulation frequencies. At $1280 \mathrm{~Hz}$ the function is essentially flat and no significant phase locking occurs. The thin horizontal line in each graph indicates a value of 3 that was selected as threshold criterion indicating significant phase-locking. Fig. 5f shows a plot of the threshold depth of modulation in relation to the modulation frequency, i.e., the temporal modulation transfer function of this cell.

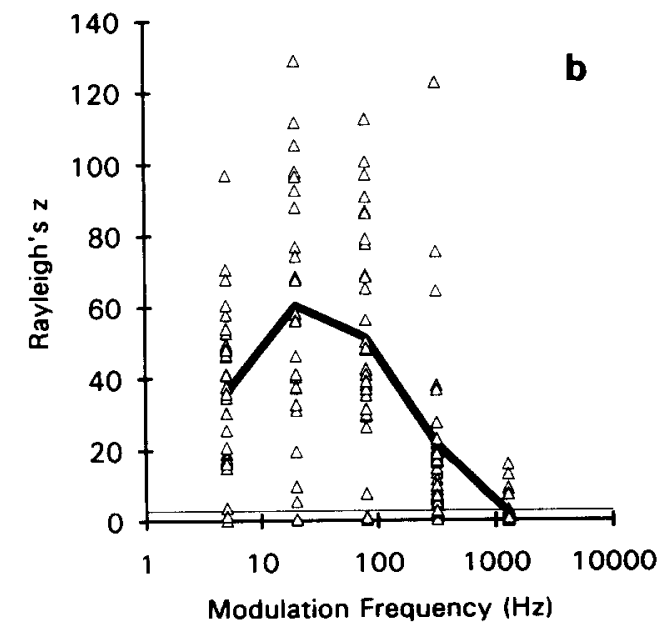

Fig. 4. (a) Vector strength and (b) Rayleighs' $z$ for the 30 units presented with the continuos stimulus at a depth of modulation of $100 \%$. The heavy continuous lines show the mean data. The thin hoizontal line in (b) indicates the amplitude modulation phase-locking threshold criterion for a significance level of $5 \%$. 

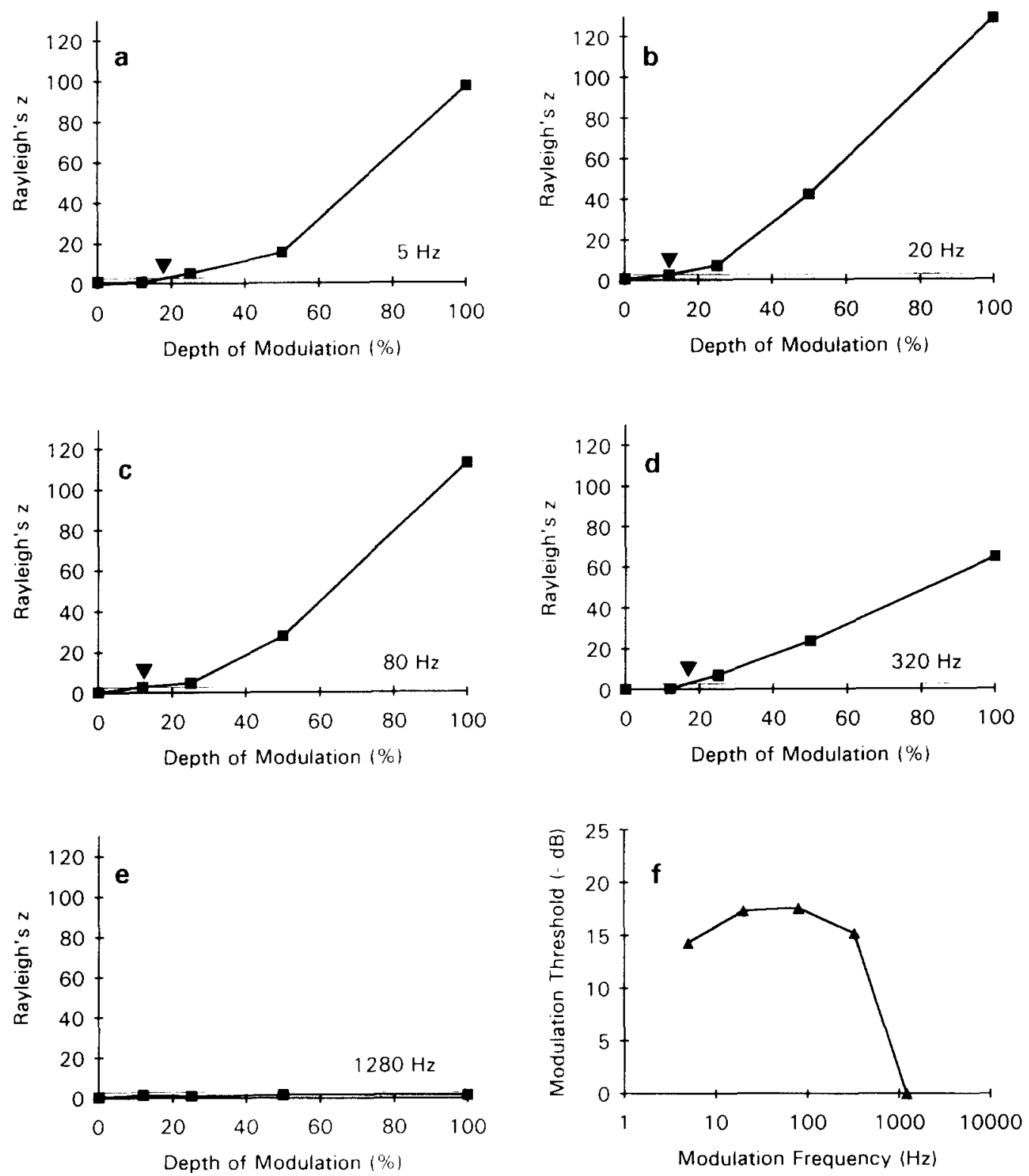

Fig. 5. Rayleighs' $\mathrm{z}$ in relation to the depth of modulation $(\mathrm{a}-\mathrm{e})$ as determined from the period histograms illustrated in Fig. 3 . The thin horizontal lines indicate the amplitude modulation phase-locking threshold criterion for a significance level of $5 \%$. The arrows point at the minimum-detectable depth of modulation as defined by the threshold criterion. In (f) the TMTF resulting from (a)-(e) is shown for this cell which is a plot of threshold depth of modulation in relation to the modulation frequency. The data are from the same neuron as in Fig. 2.

All available modulation thresholds are shown in Fig. 6 together with the averaged temporal modulation transfer functions (heavy lines) for all cells tested with the continuous (a) or the pulsed (b) stimuli. Despite the large variability among cells the average TMTFs show a band-pass characteristic with a maximum around modulation frequencies of $20-80 \mathrm{~Hz}$. Most individual TMTFs also show this bandpass characteristic (see also the gain functions in Fig. 8 below). A comparison of the averaged TMTFs for the continuous and the pulsed stimulus condition indicates systematic differences in the detection of the modulation at modulation frequencies of 5 and $20 \mathrm{~Hz}$. In the pulsed stimulus condition modulation threshold at $5 \mathrm{~Hz}$ is higher than for the continuous stimulus, while it is apparently lower for the pulsed stimulus at a modulation frequency of $20 \mathrm{~Hz}$. These differences in modulation thresholds at 5 and $20 \mathrm{~Hz}$ were significant in the cells that were tested with the pulsed as well as with the continuous stimuli (Wilcoxon matched-pairs signed-ranks test, $\mathrm{n}=23, \mathrm{p}<0.05$ ). Thus the band-pass characteristic is more pronounced for the pulsed than for the continuous stimulus.

From the individual TMTFs in response to continuous stimuli we calculated the minimum integration times of the respective cells. The minimum integration 
times of 30 cells tested with continuous stimuli had a range of 0.25 to $15.9 \mathrm{~ms}$. The cell with the integration time of $15.9 \mathrm{~ms}$ is the only one that showed significant phase locking exclusively for the $5-\mathrm{Hz}$ modulation, and it is one of those cells where no data of the tuning characteristics are available. Without this one outlier the range of minimum integration times was $0.25-3.5$ ms with a mean of $1.14 \pm 0.86 \mathrm{~ms}$ and a median of 0.97 ms. Possible relations between the minimum-integration time for cells when presented with the continuous stimuli and other response characteristics of the respective cells were tested for significant correlations by calculating Kendalls rank-correlation coefficients. There was no significant correlation between the minimum integration time and the CFs of the cells (Fig. 7a; $N=28, \tau=0.13, P=0.34$ ). The minimum-integration time was positively correlated with the threshold at $\mathrm{CF}$ $(N=27, \tau=-0.35, P=0.01)$ and consequently it was negatively correlated with the sensation level at $\mathrm{CF}$ (Fig. $7 \mathrm{~b} ; N=27, \tau=-0.35, P=0.01$; sensation level is defined as the difference between the level of the stimulus and the cell's threshold at $\mathrm{CF}$ ). It was positively correlated with the $Q_{10} \mathrm{~dB}$-value of the fibres (Fig. $7 \mathrm{c} ; N=27, \tau=0.29, P=0.03$ ), but was independent of the bandwidth $10 \mathrm{~dB}$ above threshold (Fig. 7d; $N=27, \tau=-0.02, P=0.88$ ). In addition, the minimum integration time was significantly correlated with the high-frequency slopes of the neural tuning curves $(N=24, \tau=0.30, P=0.04$ ), while the spontaneous discharge rate of the cells (Fig. 7 f; $N=27, \tau=0.05$, $P=0.74)$, the bandwidth $40 \mathrm{~dB}$ above threshold $(N=$ $16, \tau=-0.12, P=0.53)$, the $Q_{40} \mathrm{~dB}$-value $(N=16$, $\tau=0.23, P=0.21)$ and the low frequency slopes of the tuning curves $(N=24, \tau=-0.07, P=0.66)$ were not significantly correlated with the minimum integration time.

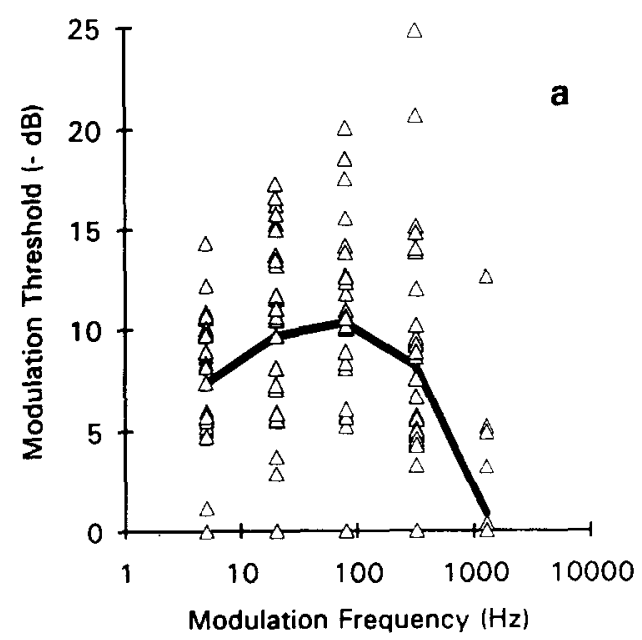

\section{Discussion}

The primary goal of this investigation was to determine the temporal resolution of primary auditory afferents in the starling with a set of amplitude-modulated stimuli that had previously been used to characterize temporal modulation transfer functions of starlings behaviourally (Klump and Okanoya, 1991). To allow a direct comparison of our neurophysiological data from the auditory nerve with those of this previous behavioural study, we applied similar methods in the analysis of the responses: modulation thresholds were determined for each modulation frequency and the TMTF was constructed by plotting these modulation thresholds versus the modulation frequencies (see Fig. 6). Since the carrier signal used in the generation of the amplitude modulated stimuli was a broadband noise, the shape of the TMTFs should only reflect the coding of the temporal pattern of the stimulus by the auditory-nerve fibres. The spectrum of the signal will remain unchanged by the amplitude modulation (see above, Fig. 1) so that no spectral cues are available to the neurons.

To our knowledge, all mammalian studies at the level of the auditory nerve used stimuli (e.g. amplitude modulated CF-tones) or methods of data analysis (e.g. gain functions; Palmer 1982; Kim et al., 1990; Joris and Yin, 1992; Frisina et al., 1990, Cooper et al., 1993; Yates, 1987) that differed from those used in this study. Despite the differences in the methodology, response patterns in the starling were similar to those found in mammals. The modulation transfer functions generally resemble a low pass filter. The roll-off frequency was below $1 \mathrm{kHz}$ in the guinea pig and the gerbil (Palmer, 1982; Frisina et al., 1990), and a slightly higher corner frequency above $1-2 \mathrm{kHz}$ was reported

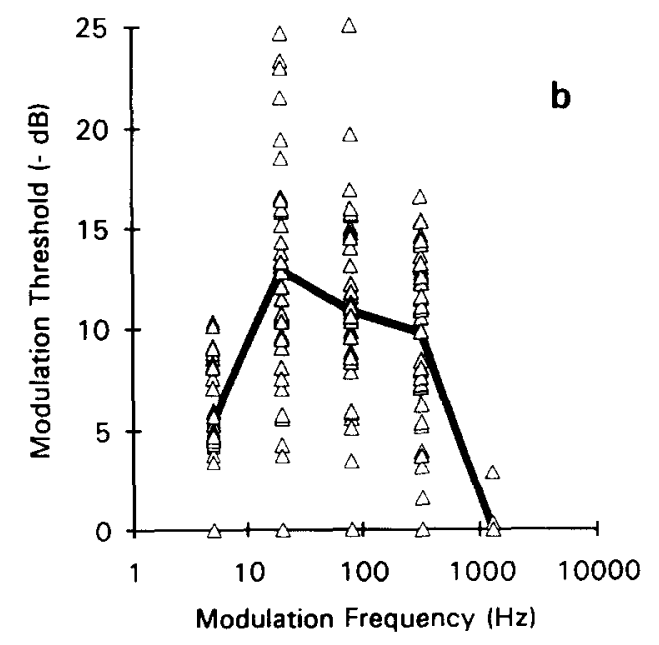

Fig. 6. Modulation thresholds in relation to the modulation frequency for continuous (a) and gated (b) stimuli. The heavy lines show the average data (continuous stimuli: $N=30$ cells; gated stimuli: $N=38$ cells). 
for the cat (Joris and Yin, 1992; Kim et al., 1990). The higher corner frequency in the cat as compared to the guinea pig and our starling data might be correlated with differences in phase locking characteristics of auditory afferents among these species (Gleich and Narins, 1988; Palmer and Russel, 1986; Johnson, 1980) which similarly may reflect limits in the coding of fast temporal changes in the signal. Similar to the results described by Joris and Yin (1992) for the cat, also in the starling the neuronal phase locking to pure tones
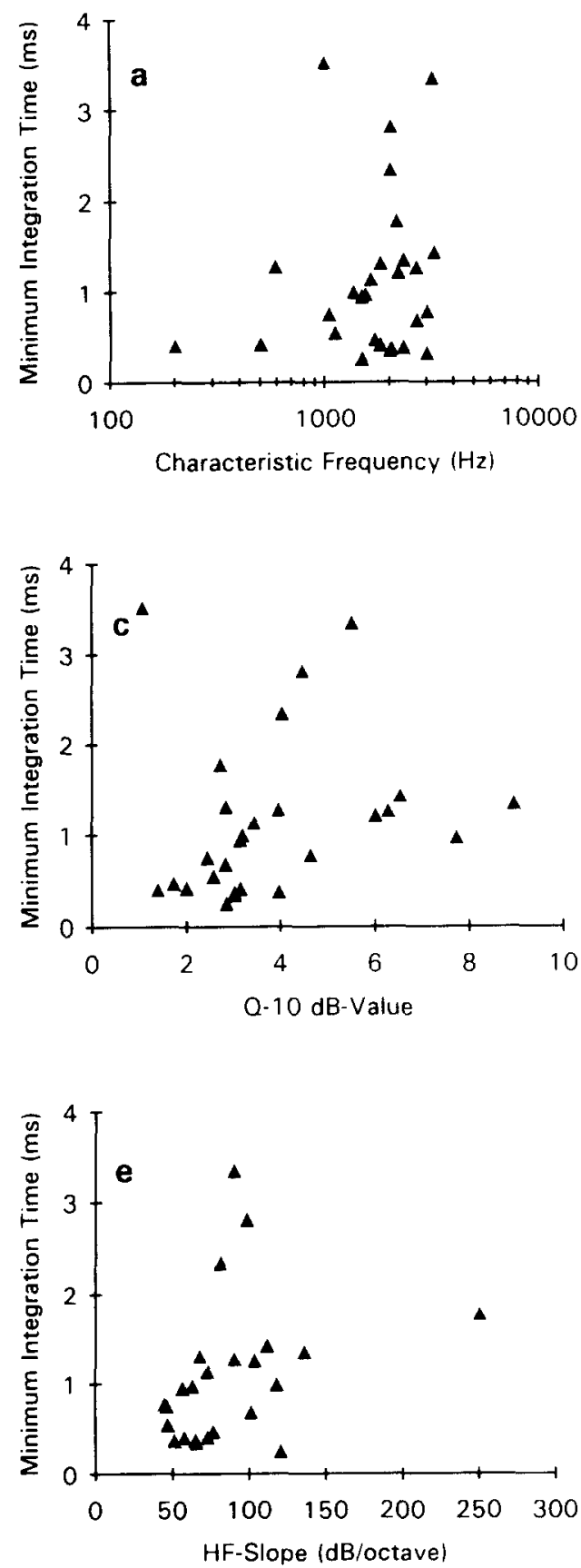

Fig. 7. The minimum integration time of the auditory-nerve fibres for the continuous stimuli in relation to the cells' $\mathrm{CF}$ (a, $N=28$ ), sensation level (b, $N=27$ ), $Q_{10} \mathrm{~dB}$-value (c, $N=27$ ), bandwidth $10 \mathrm{~dB}$ above threshold (d, $N=27$ ), high-frequency slope of the tuning curve (e, $N=27$ ), and the spontaneous rate (f, $N=27$ ).
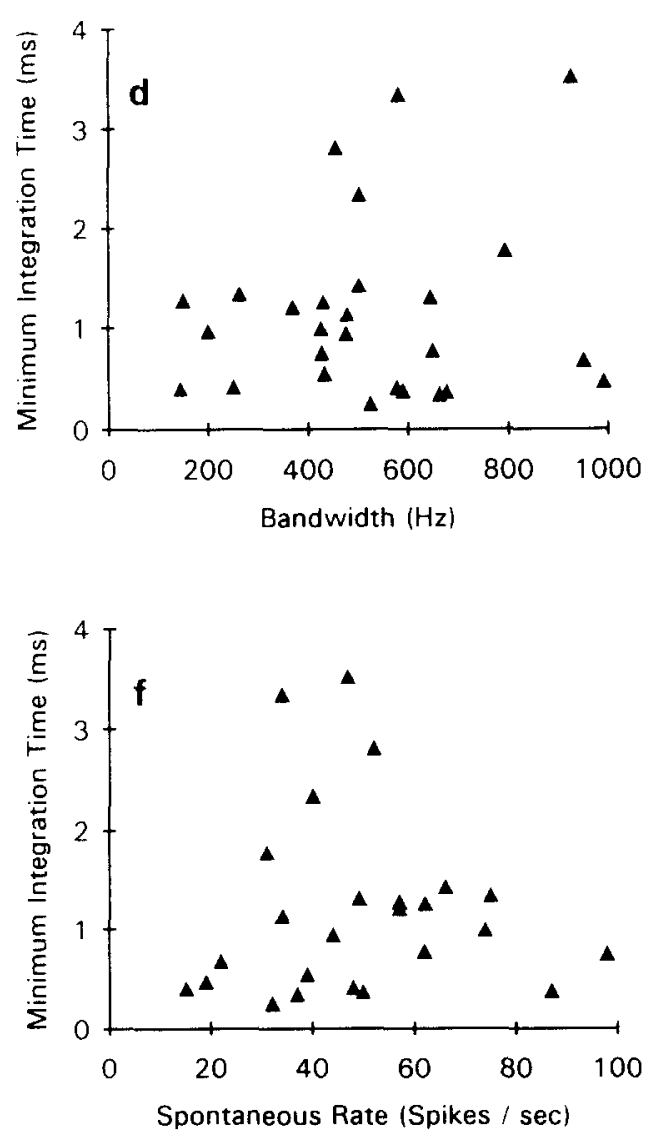

(Gleich and Narins 1988) extends to much higher frequencies than phase locking to the envelope of a modulated signal (this study). The limit of phase locking to tones was $1.4 \mathrm{kHz}$ as determined by the highfrequency cutoff of the mean vector strength $3 \mathrm{~dB}$ below its maximum (reanalysis of data shown in Fig. 4 of Gleich and Narins 1988). This is 3.2 octaves higher than the cutoff frequency of $160 \mathrm{~Hz}$ determined for phase locking to the modulation (average neural TMTF for the continuous noise stimulus). Thus, it is unlikely

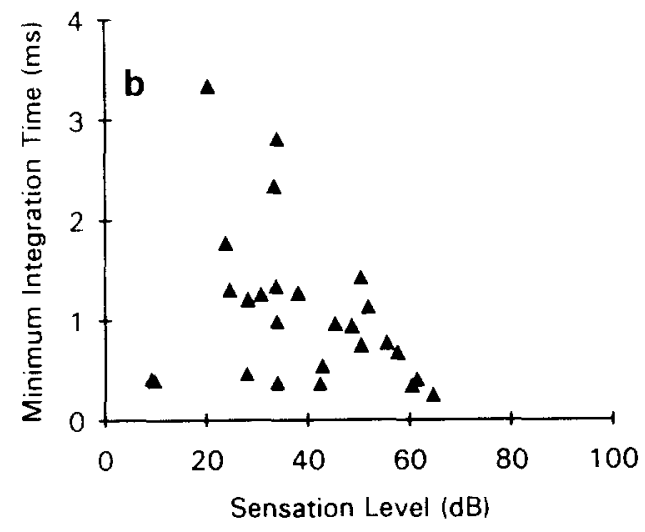




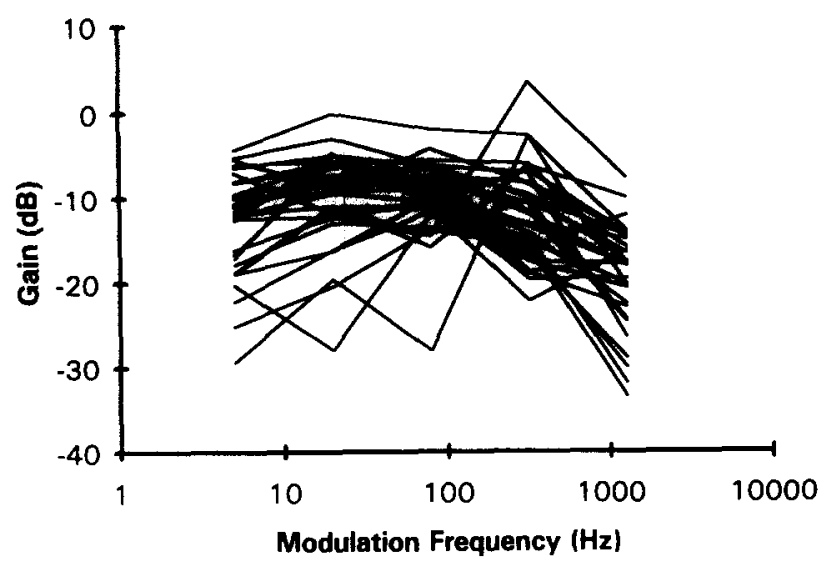

Fig. 8. The gain in the coding of modulation by auditory-nerve fibres in relation to the modulation frequency. The lines show data from 30 auditory nerve fibres. The depth of modulation was $50 \%$, and the level of the continuous noise was $75 \mathrm{~dB}$ SPL.

that mechanical properties of the cochlea limit the cutoff frequency that we found in the temporal modulation transfer functions.

The level of $75 \mathrm{~dB}$ SPL that we used in our study is relatively high considering that the coding of modulation appears to be optimal for intermediate soundpressure levels at which most I/O-functions show their steepest slopes (Langner, 1992; Joris and Yin, 1992; Cooper et al., 1993; Yates, 1987; Kim et al., 1990). For comparison with mammalian data Fig. 8 shows the starling's gain functions of auditory-nerve fibres for a depth of modulation of $50 \%$. The gain was calculated as $\mathrm{g}=20 \log (2 * \mathrm{VS} / 0.01 \mathrm{~m})$ with VS being the vector strength and $\mathrm{m}$ the modulation depth in\% (Langner, 1992). Only few cells had a gain that was better than $-5 \mathrm{~dB}$, while the average gain was -10 to $-15 \mathrm{~dB}$ for the range of modulation frequencies between 5 and $320 \mathrm{~Hz}$. These observations are very similar to mammalian results obtained at high stimulus levels (Frisina et al., 1990, Kim et al., 1990).

A study at the level of the auditory nerve that used sinusoidally amplitude-modulated noise and, comparably to our analysis, constructed TMTFs from the modulation thresholds at different modulation frequencies was conducted in a neotropical tree frog (Eleutherodactylus coqui, Dunia and Narins, 1989). As found in the starling, most TMTFs in the auditory-nerve fibres of this frog showed a low-pass characteristic when measured with a continuous noise carrier. However, the high frequency slopes of the TMTFs in the coqui frog reported as roughly $20 \mathrm{~dB} /$ octave were steeper than the slopes of approximately $4 \mathrm{~dB}$ /octave that we can derive from the averaged TMTFs of the starling (Fig. 6). The low high frequency slopes in the starling could have been affected by the fact that the modulation thresholds were only determined in 2-octave frequency steps between modulation frequencies of 5 to
$1280 \mathrm{~Hz}$. Thus, steeper high-frequency slopes of the TMTFs could have remained undetected between 320 and $1280 \mathrm{~Hz}$. However, the similarity in the shapes of our average neural TMTFs with those determined behaviourally with twice the modulation-frequency resolution (Klump and Okanoya, 1991) suggests that the lower high frequency slopes of the TMTFs in the starling may be real. The high-frequency roll-off of the TMTF determined for the starling's auditory-nerve fibres is also very similar to the high-frequency roll-off in other behavioural studies in humans (Viemeister, 1979; Forrest and Green, 1987; Formby and Muir, 1988), chinchilla (Salvi et al., 1982) and the parakeet (Dooling and Searcy, 1981).

In the coqui frog minimum integration times covered a range of between 0.17 and $2.9 \mathrm{~ms}$ (Dunia and Narins, 1989) and were on average well below those we found in starlings. The median of $0.97 \mathrm{~ms}$ in the starling is more than twice the median of $0.42 \mathrm{~ms}$ reported for the coqui frog. In our sample only $30 \%$ of the cells had minimum integration times below $0.5 \mathrm{~ms}$ (corresponding to a cut-off frequency of $320 \mathrm{~Hz}$ ) as compared to $66 \%$ in the frog. One factor that could contribute to the difference in the minimum integration times between the coqui frog and the starling might be related to differences between the two species in the frequency selectivity of auditory afferents. In the coqui frog less than $20 \%$ of the fibres had $Q_{10} \mathrm{~dB}$-values above 2.0 as compared to more than $80 \%$ in the starling. In addition all starling fibres had bandwidths $10 \mathrm{~dB}$ above threshold that were below $1 \mathrm{kHz}$ while in more than one third of the frog fibres the bandwidth was above $1 \mathrm{kHz}$ (Dunia and Narins, 1989). Fay (1980) reports that in the goldfish many saccular nerve fibres show a remarkable good detection of amplitude modulation of a noise carrier even at the highest modulation frequency tested $(400 \mathrm{~Hz})$. Although this study reports no minimum integration times, the gold fish TMTFs indicate that they would be rather short (i.e. below 0.5 $\mathrm{ms})$. In addition these sacuular nerve fibres are generally broadly tuned and have $Q_{10} \mathrm{~dB}$ values below 1 . Thus the higher frequency-selectivity in the starling cochlea could have contributed to the larger minimum integration times as compared to those found in the coqui frog and probably also in the goldfish (see also discussion below).

In general, frequency selectivity and temporal resolution of a filter show an inverse relationship. In contrast to the expectation based on the assumption of a simple proportionality, we did not find a significant correlation between the minimum integration time and the bandwidth of the fibers or its reciprocal. We also tried to correlate 3 other measures of frequency selectivity $\left(Q_{10} \mathrm{~dB}\right.$-value and the low- and high-frequency slopes of the tuning curves) with the minimum integration time. Although we found that the minimum inte- 
gration times were significantly correlated with the $Q_{10}$ $\mathrm{dB}$-value and the high-frequency slope of the tuning curves, the scatter in the data was quite high (Fig. 7) and the correlation coefficients were rather low. They can only explain about $15-20 \%$ of the variance in the minimum integration time $\left(\mathrm{r}_{\mathrm{s}}=0.39\right.$ and 0.45 for $Q_{10}$ $\mathrm{dB}$-value and high frequency slope, respectively). The relation between tuning related measures and the temporal resolution is quite weak and only some of the correlations in our comparatively small sample of cells reached significance. A similar discrepancy between the theoretical expectations and the experimental observations was also found in the frog (Dunia and Narins, 1989) where the variation in frequency selectivity explained only about $5 \%$ of the variation in the temporal resolution. The present starling data are also consistent with results from a previous study in which temporal resolution in the starling was measured with a gap-detection paradigm (Klump and Gleich, 1991). Also in this study we found no significant relations between the minimum-detectable gap and the bandwidth $10 \mathrm{~dB}$ above threshold or the $Q_{10} \mathrm{~dB}$-value.

According to theory, the time constant of a filter $(\tau)$ that describes its temporal resolution is proportional to the reciprocal of a filter's bandwidth (BW), i.e., $\tau=$ $\mathrm{k} * 1 / \mathrm{BW}$. For the higher-order filters necessary to explain the sharp tuning of peripheral auditory filters de Boer (1985) estimates that the constant $\mathrm{k}$ ranges from 1.3 to 4.2 compared to $\mathrm{k}$ being equal to $1 / \mathrm{p}$ for a single resonance. Thus, for a given bandwidth of a filter the temporal resolution may vary over a wide range depending on the complexity of the filter (e.g., see de Boer and Kruidenier, 1990). The variation in the complexity of peripheral filters is also evident in the starling. At a given CF, the measures that are related to the frequency selectivity of starling's auditory-nerve fibres, e.g., the $Q_{10} \mathrm{~dB}$-values and the high- and low-frequency slopes of the tuning curves, vary considerably (Manley et al., 1985). Such variability may reduce the correlation between the minimum integration time and the bandwidth of the tuning curves in a population of fibers (Klump and Gleich, 1991).

Our observations that the minimum integration times are significantly correlated with the neural threshold at $\mathrm{CF}$ and the sensation level of the stimulus while $\mathrm{CF}$ and spontaneous discharge rate show no significant correlation agree with the results previously obtained in a study of temporal resolution in starlings using a gap-detection paradigm (Klump and Gleich, 1991).

There is a close correspondence between the temporal modulation transfer functions measured in the auditory nerve and those determined in a psychoacoustical study in the starling (Fig. 9). While the minimum depth of modulation needed for the detection differs between the average auditory-nerve response and the behavioural temporal modulation transfer function (although a few fibres were as sensitive as the whole animal), the general shape of the function is the same when measured both physiologically and behaviourally. The transition in the shape of the temporal modulation transfer function from a lowpass to a more bandpasslike characteristic which was observed in the psychoacoustic study comparing the gated with the continuous stimulus presentation (Klump and Okanoya, 1991) can also be found in the average neuron's response. A closer view at our data suggests, that this difference is mainly due to the neurons' onset-response when presented with the gated signal. If we delay our $600-\mathrm{ms}$ analysis window so that it starts $100 \mathrm{~ms}$ after the stimulus onset rather than at the onset, the difference in the shape of the temporal modulation transfer function observed between the gated and the continuous stimulus condition disappears. This suggests that at some modulation frequencies the phasic onset re-
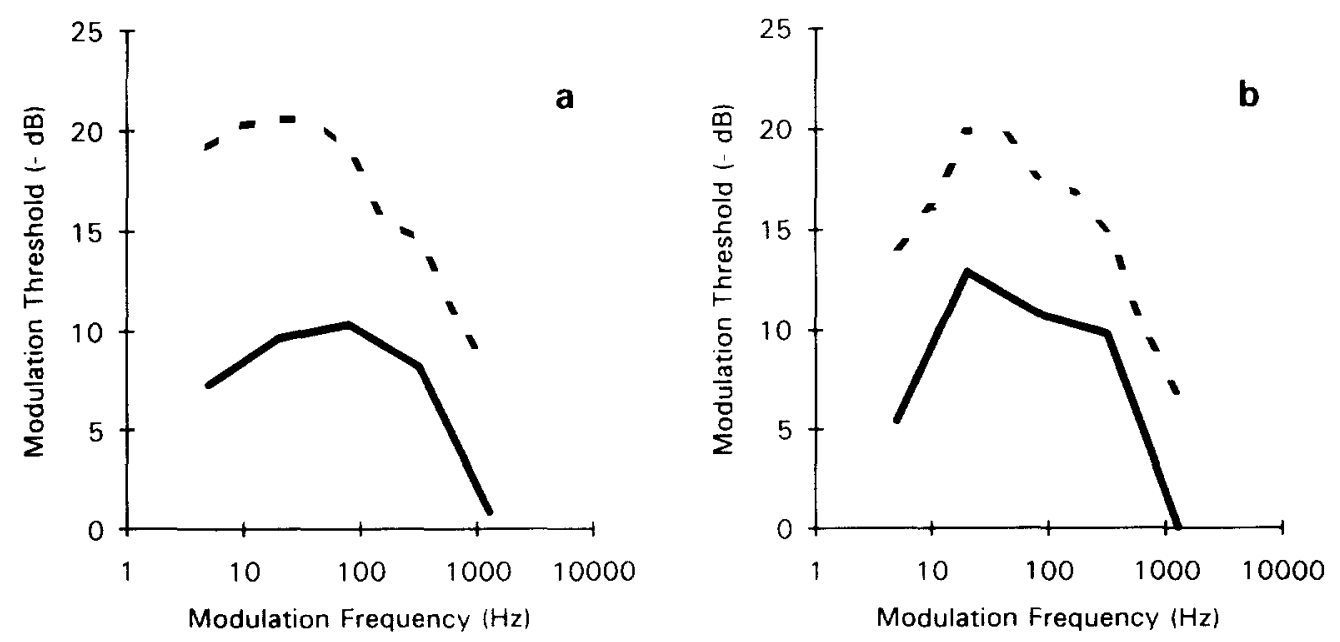

Fig. 9. A comparison between the average neural (solid line) and the behavioural (Klump and Okanoya 1991, dashed line) temporal modulation transfer function of the starling: (a) continuous noise, (b) gated noise. 
sponse enhanced the period pattern of the modulation (e.g., at a modulation frequency of $20 \mathrm{~Hz}$ ) while at other modulation frequencies it reduced the average synchronization of the neuron's response to the modulation (e.g., at a modulation frequency of $5 \mathrm{~Hz}$ ). The effects of adaptation may also be responsible for the differences in the shape of the TMTF for gated and continuous carriers that was observed in other studies (e.g., in a psychoacoustic study in humans by Viemeister, 1979, and in a study in the goldfish, see Coombs and Fay 1985).

The minimum integration times that can be derived from the neuronal and the behavioural temporal modulation transfer functions were 1.0 and $1.3 \mathrm{~ms}$, respectively (comparing the median of the neuronal data with the average behavioural response for a broad-band carrier). Thus, for temporal modulation transfer functions there is a close correspondence between the average neuronal and the behaviourally determined minimum integration time, whereas in the gap-detection paradigm the median minimum-detectable gap is much larger than the starlings behavioural gap-detection threshold (12.8 ms versus $1.8 \mathrm{~ms}$ comparing the median data from primary auditory neurons with the birds psychoacoustic data, see Klump and Gleich, 1991). In psychoacoustic studies in the starling, the ratio between the gap-detection threshold $(1.8 \mathrm{~ms})$ and the minimum integration time $(1.3 \mathrm{~ms})$ was about 1.4 (Klump and Maier 1989, Klump and Okanoya 1991). This ratio is quite different when we look at the median responses of the peripheral neurons (for these, the ratio is 13.2 , i.e., a magnitude larger than that observed in the behaviour).

It has been suggested that there exists an inverse relationship between the minimum integration time and threshold depth of modulation (Dooling and Searcy, 1981; Dunia and Narins, 1989), although no underlying mechanism has been specified. Our data do not seem to fit into this scheme. The best modulation thresholds of the starling's neuronal temporal modulation transfer functions varied between 6 and 50\% depth of modulation with an average of $21 \%$ (corresponding to $-13.5 \mathrm{~dB}$, see Fig. 6). Dunia and Narins (1989) report threshold depths of modulation between 10 and $20 \%$ for coqui frog auditory nerve fibres. These frog fibre thresholds are lower than the values we found in the starling (i.e., more sensitive). However, in contrast to the predicted trade-off the minimum integration times of the frog fibres are well below those of the starling. Similar deviations from the suggested trade-off between minimum integration time and the minimum detectable modulation are also apparent in behavioural data. In the starling the behaviourally determined minimum detectable modulation is near $9 \%$ (i.e., -20.9 dB, Klump and Okanoya, 1991) which is very similar to findings in the parakeet (Dooling and Searcy, 1981) and the chinchilla (Salvi et al., 1982). Despite the similarity in minimum detectable modulation, the minimum integration time in the chinchilla is shorter $(0.8$ $\mathrm{ms})$ as compared to starling $(1.2 \mathrm{~ms})$ and parakeet $(1.3$ $\mathrm{ms})$. Finally, the comparison of the starling's neuronal temporal modulation transfer functions with the behavioural functions also suggests a weak relationship between both measures. Although the starling's average neuronal threshold depth of modulation is about $10 \mathrm{~dB}$ less sensitive than the behavioural threshold, the minimum integration times are about the same.

Our results demonstrate that in the starling the temporal resolution measured by the temporal modulation transfer functions is very similar when the responses at the level of the auditory nerve are compared with the performance of the animal in a behavioural test (Klump and Okanoya 1991). This is in accordance with results from an earlier study on temporal resolution in the starling's auditory system using a gap-detection paradigm which showed that the most sensitive peripheral neurons could explain the limits of temporal resolution determined behaviorally (Klump and Maier 1989, Klump and Gleich 1991). These observations suggests, that the temporal resolution in the starling's auditory system may be limited by the response properties of the auditory-nerve fibres.

\section{Acknowledgements}

This study was supported by a grant from the Deutsche Forschungsgemeinschaft within the SFB 204 'Gehör' and a Helmholtz grant by the BMFT to OG. We thank P.M. Narins for his comments on a previous version of the manuscript.

\section{References}

Buchfellner, E., Leppelsack, H.-J., Klump, G.M. and Häusler, U. (1989) Gap-detection in the starling (Sturnus vulgaris): II: Coding of gaps by forebrain neurons. J. Comp. Physiol. A 164, 539-549.

Cooper, N.P., Robertson, D. and Yates, G.K. (1993) Cochlear nerve fiber responses to amplitude-modulated stimuli: Variations with spontaneous rate and other response characteristics. J. Neurophys. 70, 370-386.

de Boer, E. (1985) Auditory time constants: A paradox? In: A. Michelsen (Ed.), Time resolution in auditory systems. Springer Verlag, Berlin, Heidelberg, New York, pp. 141-158.

de Boer, E. and Kruidenier, C. (1990) On ringing limits of the auditory periphery. Biol. Cybern. 63, 433-442.

Coombs, S. and Fay, R.R. (1985) Adaptation effects on amplitude modulation detection: Behavioral and neurophysiological assessment in the goldfish auditory system. Hear. Res. 19, 57-71.

Dooling, R.J. and Searcy, M.H. (1981) Amplitude modulation thresholds for the parakeet (Melopsittacus undulatus). J. Comp. Physiol. $143,383-388$.

Dunia, R. and Narins, P.M. (1989) Temporal resolution in frog auditory-nerve fibers. J. Acoust. Soc. Am. 85, 1630-1638. 
Fastl, H., Hesse, A., Schorer, E., Urbas, J. and Müller-Preuss, P. (1986) Searching for neural correlates of the hearing sensation fluctuation strength in the auditory cortex of quirrel monkeys. Hear. Res. 23, 199-203.

Fay, R.R. (1980) Psychophysics and neurophysiology of temporal factors in hearing by the goldfish: Amplitude modulation detection. J. Neurophys. 44, 312-332.

Fay, R.R. (1985) Sound intensity processing by the goldfish. J. Acoust. Soc. Am. 78, 1296-1309.

Formby, C. and Muir, K. (1988) Modulation and gap detection for broadband and filtered noise signals. J. Acoust. Soc. Am. 84, $545-550$.

Forrest, T.G. and Green, D.M. (1987) Detection of partially filled gaps in noise and the temporal modulation transfer function. $J$. Acoust. Soc. Am. 82, 1933-1943.

Frisina, R.D., Smith, R.L. and Chamberlain, S.C. (1990) Encoding of amplitude modulation in the gerbil cochlear nucleus: I. A hierarchy of enhancement. Hear. Res. 44, 99-122.

Giraudi, D., Salvi, R., Henderson, D. and Hamerik, R. (1980) Gap detection by the chinchilla. J. Acoust. Soc. Am. 78, 1296-1309.

Gleich, O. (1994) Excitation patterns in the starling cochlea: A population study of primary auditory afferents. J. Acoust. Soc. Am. 95, 401-409.

Gleich, O. and Narins, P.M. (1988) The phase response of primary auditory afferents in a songbird (Sturnus vulgaris L.) Hear. Res. 32, 81-92.

Henderson, D., Salvi, R., Pavec, G. and Hamerik, R. (1984) Amplitude-modulation thresholds in chinchillas with high-frequency hearing loss. J. Acoust. Soc. Am. 95, 401-409.

Johnson, D.H. (1980) The relationship between spike rate and synchrony in responses of auditory nerve fibres to single tones. J. Acoust. Soc. Am. 68, 1115-1122.

Joris, P.X. and Yin, T.C.T. (1992) Responses to amplitude-modulated tones in the auditory nerve of the cat. J. Acoust. Soc. Am. $91,215-232$.

Kim, D.O., Sirianni, J.G. and Chang, S.O. (1990) Responses of DCN-PVCN neurons and auditory nerve fibers in unanesthetized decerebrate cats to AM and pure tones: Analysis with autocorrelation/power-spectrum. Hear. Res. 45, 95-113.

Klump G.M. and Maier E. (1989) Gap detection in the European starling (Sturnus vulgaris). I. Psychophysical thresholds. J. Comp. Physiol. A 164, 531-538.

Klump G.M. and Gleich O. (1991) Gap detection in the European starling (Sturnus vulgaris). III. Processing in the peripheral auditory system. J. Comp. Physiol. A 168, 469-476.
Klump G.M. and Okanoya K. (1991) Temporal modulation transfer functions in the European Starling (Sturnus vulgaris): I. Psychophysical modulation detection thresholds. Hear. Res. 52, 1-12.

Langner, G. (1992) Periodicity coding in the auditory system. Hear. Res. 60, 115-142.

Manley, G.A., Gleich, O., Leppelsack, H.-J. and Oeckinghaus, H. (1985) Activity patterns of cochlear ganglion neurons in the starling. J. Comp. Physiol. A 157, 161-181.

Palmer, A.R. (1982) Encoding of rapid amplitude fluctuations by cochlear nerve fibres in the guinea pig. Arch. Otorhinolaryngol. 236, 197-202.

Palmer, A. (1990). The representation of the spectra and the fundamental frequencies of steady-state single- and double-vowel sounds in the temporal discharge patterns of guinea pig cochlear-nerve fibers. J. Acoust. Soc. Am. 88, 1412-1426.

Palmer, A.R. and Russel, I.J. (1986) Phase-locking in the cochlear nerve of the guinea-pig and its relation to the receptor potential of inner hair-cells. Hear. Res. 24, 1-15.

Ronacher, B. and Römer, H. (1985) Spike synchronization of tympanic receptor fibres in a grasshopper (Chorthippus biguttulus $\mathbf{L}$., Acrididae). A possible mechanism for the detection of gaps in model songs. J. Comp. Physiol. A 157, 631-642.

Salvi, R.J., Giraudi, D.M., Henderson, D. and Hamerik, R.P. (1982) Detection of sinusoidal amplitude modulated noise by the chinchilla. J. Acoust. Soc. Am. 71, 424-429.

Shailer, M.J. and Moore, B.C.J. (1983) Gap detection as a function of frequency, bandwidth and level. J. Acoust. Soc. Am. 74, 467-473.

Surlykke, A., Larsen, O. and Michelsen, A. (1988) Temporal coding in the auditory receptor of the moth ear. J. Comp. Physiol. A 162, 367-374.

Viemeister, N.F. (1979) Temporal modulation transfer function based upon modulation thresholds. J. Acoust. Soc. Am. 66, 1364-1380.

Winter, I.M. and Palmer, A.R. (1990). Temporal responses of primary-like anteroventral cochlear nucleus units to the steady-state vowel /i/. J. Acoust. Soc. Am. 88, 1437-1441.

Yates, G.K. (1987) Dynamic effects in the input/output relationship of auditory nerve. Hear. Res. 27, 221-230.

Zar H.H. (1984) Biostatistical analysis. Prentice-Hall Inc., Englewood Cliffs, New Jersey.

Zhang W., Salvi R.J. and Saunders S.S. (1990) Neural correlates of gap detection in auditory nerve fibres of the chinchilla. Hear. Res. 46, 181-200. 